\title{
A Review of Research on Detection and Evaluation of the Rail Surface Defects
}

\section{Lei Kou}

Institute of Railway Systems and Public Transport, TU-Dresden, Reitbahnstr. 35, 01069 Dresden, Germany; lei.kou@tu-dresden.de

\begin{abstract}
Defects on the rail surface will hasten the wear of the wheels. At the same time, when the wheel is periodically hitting and rolling surface defected, the defects will gradually develop into the interior, which significantly increases the possibility of a train derailment and serious safety accidents. A timely checking of the railway tracks to find defects as early as possible is a basic condition for ensuring the safety of railway operations. It also prolongs the service life of railways because most of the rolling contact fatigue (RCF) can be eliminated during rail grinding. Such defects appear as spalling and cracks in the first stage of the rail surface. Manual detection has been challenging to meet the large-scale railway running mileage. Therefore, a more efficient automatic detection method is indispensable. This article reviews the latest research and exploration on the defect inspection of rail surfaces in recent years. In the article, not only the application of traditional ultrasonic and acceleration detection methods but also contributing computer vision and deep learning to detect defects on the rail surface. The new detection technology can even classify and evaluate the damage, further improving the efficiency of the detection system. Besides, the emerging research on defect state prediction to reduce inspection costs is interesting.
\end{abstract}

Keywords: rail surface defects; machine learning; detect; $C N N$

\section{Introduction}

In the $21^{\text {st }}$ Century, railway transport will be an increasingly important task related to the developed world and society. It started in the $19^{\text {th }}$ and $20^{\text {th }}$ Centuries with enormous railway construction procedures worldwide [1-3]. Railway construction investment has been continually increasing. It can be said that traffic safety is an important consideration for traffic users when choosing a particular means of transport. It is critical to the scale of traffic implementation, revenue, and overall business performance [4]. The total railway mileage in the world is about 1.5 million kilometers, and the European Union accounts for about 25\% [5]. The rail is an important part of the railway. The defects on the surface of the rails have caused great damage to the wheelsets and bearings of the rail vehicles. When the 
wheels are moving on the track with defective surfaces, periodic impacts will cause the coupled vibration of the entire vehicle and the track system. It will reduce the service life of the train components and a significant cause of vehicle derailment overturning and combustion axles. Therefore, defect detection of railway tracks is an essential means to ensure the safety of railway transportation. The annual railway inspection cost in the EU (European Union) alone is about 70 million euros [6]. Finding rolling contact fatigue (RCF) as early as possible cannot only avoid traffic accidents but also take timely measures to extend the service life of rails [7], both of which can reduce railway running costs [8]. The detection of track surface defects has long relied on manual inspections. This detection method needs experts to visually inspect components and use specific tools to identify any defects in the rail surface [9]. However, this method is cumbersome, hardworking, and prone to human error. In addition, the personal safety of the inspectors is also an issue that needs to heed. With the increase in demand for high-speed railway transport and advanced technology, it is necessary to develop an automatic intelligent detection system to replace manual detection in detecting track surface defects.

Although the continual improvement of technology has reduced the probability of internal defects in the rail, the probability of fatigue cracks on the surface of the rail is still high [10]; harsh environments can also cause surface defects, which may irritate worsening the rail $[11,12]$. Surface defects of rail can be divided into the wave-shaped abrasion and discrete disease of the rail. Wave-shaped abrasion refers to the irregularity of the wavy shape of the periodic disease that appears on the surface of the rail in the longitudinal direction [13]. Discrete damage occurs randomly on the surface of the rail [14]. With developing technology, people have fully researched and applied the detection methods of rail surface defects by using the above technologies. Non-contact detection methods based on machine vision have gradually developed and been widely used in electrical, electronic, mechanical, automotive, and industrial inspections. Machine learning can even predict some regular damage.

This article reviewed the rail surface defect detection, recognition, classification, and evaluation methods proposed and improved in the past ten years. Technical analysis, the characteristics of technology, cost performance, accuracy, and the object to which the method is applicable. However, the results of each method and the matching limits are recorded. Through comparing and analysis methods that can evaluate the size or severity of rail surface defects or classify them to select the best rail surface damage detection method. The organization of this article is as follows: Chapter 2 expounds on the research results of detection and recognition, discusses and emphasizes the advantages and limits of each method in the paper. Chapter 3 introduces the research results of classification, evaluation, highlights the accuracy and background conditions of every method in the article. Chapter 4 discusses the results, analyzes the characteristics of the method, and shows the overall progress in the field. Finally, Chapter 5 summarizes the purpose and results of this article, and prospects for detecting technologies. 


\section{Detection Methods}

In the paper, the detection methods shown in 70 research papers regained by Scopus can be divided into six categories: geometry measuring, detecting wheel track motion (vibration or acceleration), using electric or magnetic field changes, using acoustic or light waves (thermal sensors), Image processing and machine learning. The research field is mainly concentrated in the emerging image processing and machine learning fields. Because other detection methods have been around for a long-time and have been thoroughly studied, the new research mainly explores improving detection technology and optimizes a particular step. In this section, a comprehensive review of these papers will be conducted. Analyze the performance, results, and limits of each technology.

\subsection{Geometric Measurement}

Although measuring geometric information can get the situation of the railway track through many indicators [15], good results have been achieved. A single geometric measurement method is used to obtain the geometric data of the track surface, mainly through a mechanical stylus or laser reflection. These methods can only detect a cross-section and cannot provide macroscopic inspection data. Later manual inspections are still required to confirm the location and size of surface defects, but most inspections in the railway industry do not use $3 \mathrm{D}$ reconstruction technology. To complete 3D technology combines cross-sectional data with a longitudinal plane positioning based on geometric detection, making 3D model reconstruction possible in defect detection on the rail surface. Ye et al. present a novel 3D perceptual system based on a low-cost 2D laser sensor [16]. This method uses the 2D laser sensor detection system and a drawing sensor to decide the longitudinal position movement. The data are formed into a three-dimensional point set. 3D modeling by Matlab. Use the 3D model to identify rail surface defects. Rikhotso et al. also applied this method to measure rail profile and detect defects on the track surface [17]. Casey Jessop and Naeimi each proposed a 3D modeling method combining the stylus Profile-metric and X-Ray scan data. The results showed a geometry similar to the squat network, also possible to infer the crack tip of the surface to a deeper depth that needs improvement. Still, the description of the mid-depth squat crack network is sufficiently accurate $[18,19]$.

The above methods can theoretically detect surface defects, but they also have obvious shortcomings. Radiography is mainly used to detect internal defects. Surface defects are just derived properties and cannot be quickly moved to detect. The geometric detection of mechanical contacts also has this disadvantage. Laser sensors are sensitive to the environment, too strong light will affect the measurement results, and strenuous exercise will cause high deviations. Niu et al. proposed a method based on a binocular line scanning system, which can obtain contour information in the high-precision image while avoiding the decoding 
distortion of the structured light reconstruction method [20]. Sysyn et al. applied the modeling method to detect surface wear of turnouts, using the measurement data on the surface scanning device to establish the 3D model of the turnouts and analyze the change of the wheel/rail trajectory to detect the defect of turnouts [21]. This method has high 3D modeling accuracy while high detection speed, which is a research direction worth looking forward to.

\subsection{Electromagnetic Detection}

Electromagnetic detection is a broad region of technologies used in railways. Due to surface inspection of blind subjects in conventional ultrasonic technology, an eddy inspection method based on electromagnetic principles has been proposed to detect the rail surface and near-surface defects [22]. Eddy testing uses alternating magnetic fields to create vortex-like induced alternating currents in the measured conductive Rail. The conductivity, magnetic permeability, defects, defect size, and shape of the measured part affect the distribution and size of the eddy. Through the coil-detection to measure the magnetic field change caused by the eddy. The distribution, size, and phase of the eddy in the test piece were measured without contact. It can perform high-speed inspections but has a skin effect and can only detect the surface and near-surface structure status of conducive materials [23]. The eddy testing technology has matured application examples in Eurailscout in Germany and Sperry in the United States [24]. However, lift-off has a more significant impact on the accuracy of rapid inspection. Huang and others, the Chinese Academy of Railway Sciences, and others have studied the card-type eddy sensor used to detect surface defects of curved turnout rails [25]. The research team of Nanjing University of Aeronautics and Astronautics has been continuously researching magnetic flux leakage detection technology in recent years. The team studied the development of array-type magnetic flux leakage inspection equipment and quantitative analysis of rail cracks to get defect location and distribution characteristics. Under the simulated conditions of the laboratory, the inspection speed of cracks with a width of $0.2 \mathrm{~mm}$ can reach up to $200 \mathrm{~km}$ per hour, which is in the leading position in the field [26]. This team added a ferrite in a sensor to reduce the reluctance to increase the magnetic intensity above the defects to increase the MFL signals [27]. It also gives an improved adaptive filtering method is proposed to solve the problem caused by filtering the MFL signal on the rail top surface [28].

The alternating magnetic field measurement method detects material defects by measuring the changes in the induced magnetic field on the surface of the Rail, which can realize the accurate positioning and measurement of defects. The data Rows Handel collects from ACFM sensors are processed offline by combining threshold and feature matching methods rather than simple threshold methods. Even in the presence of peeling changes and noise, the automatic detection of surface damage defects has high reliability [29]. Chacón Muñoz et al. presents the 
B-Spline approach used for the accurate filtering of the noise of the raw ACFM signal gained during high-speed tests to improve the reliability of the measurements [30]. Papaeias et al. studied the applicability of ACFM technology in detecting and assessing the severity of surface cracked RCF defects [31]. ACFM technology has an extensive development prospect in detecting and quantifying RCF cracks. However, it is necessary to overcome the influence of the probe on the detection sensitivity caused by being too far from the object's surface. It is needed to continue improving the sensitivity of corrugated and polished track detection.

\subsection{Track-side and On-board Vibration and Acceleration Detection}

The dynamic interaction between the wheels and the track produces vibrations and varying accelerations transferred through the track system, air, and rolling stock. This vibration is generally used to measure rail defects in squats. Axlebox acceleration (ABA) measurement is often used to identify railway and track irregularities. The squat is a kind of rolling contact fatigue defect, and early detection is conducive to the safe operation of railways [32]. Mykola Sysyn et al. deal with measurement interpretation problems by analyzing vibration to estimate the health of the turnout [21]. Some scholars try to use this technology to detect spalling defects on the rail surface. Andrew Keong $\mathrm{Ng}$ aims to model and simulate three common defects with three degrees of damage, process and analyze the simulated RSD-driven ABA signal, and create explicit dynamic finite element models of wheel-rail interaction for tracks with and without RSDs [33]. Sysyn et al. introduced a method to detect the defect of the turnout by using the ESAH-F system to perform the machine learning on the inertial measurement data of the axle box on the running train [34]. The research team then discussed the HilbertHuang transform method used to overcome the relationship between the measured acceleration component and impact lateral distribution and the life cycle of ordinary turnout contact surface [35]. However, this detection method is challenging to use to detect cracks and small peeling. Besides, it is not practical outside the laboratory, because the vertical acceleration of the train in operation is affected by too many reasons.

\subsection{Sound and Light Wave Detection}

Acoustic wave detection uses the characteristics of sound wave reflection, diffraction, and transmission to determine whether there are defects inside the measured rail by watching the waveform, echo, sound velocity, attenuation, and resonance of the ultrasonic wave in the measured rail. Conventional ultrasonic technology has been widely applied in the detection of internal defects in steel rails. Defects extending in the horizontal direction and longitudinally close to the 
gauge angle will reflect the ultrasonic wave, and obstruct the incidence of the sound beam so that the dangerous cracks buried under it cannot be detected. There is a prominent detection blind zone, defects with a depth of less than $4 \mathrm{~mm}$ from the surface of the tested part. There will be missed inspections. Therefore, ultrasound is generally not used for surface defect detection. However, Yi Jiang et al. and Hui Zhang et al. still tried to detect near-surface damage [36, 37]. The outstanding research is that Yuehong Zhang et al. studied the technology of rapid laser ultrasonic detection of rail surface defects and designed an interlaced laser ultrasonic defect detection imaging method: laser ultrasonic signals are excited on both sides of the rail to detect, and the images are fused and registered by algorithms. Filtering and image registration, obtaining a complete rail surface inspection image, displaying defect characteristics, and solving laser ultrasonic insensitive to defects [38]. Also, Bilawal Ramzan et al. proposed a research method to use Active Thermography to detect railroads surface crack [39]. The reflection of microwaves can be used to measure distance. Andrey Zhuravlev et al. proposed using the microwave or short waves to detect the rail surface, but it has become a method [40].

\subsection{Image Processing Detection}

Accurate judgment and cognition through computer vision research have been applied in many fields with the development of science and technology. The basic principle of rail computer visual damage detection is to use an image acquisition device to obtain information on the surface of the rail and convert it into an electronic image signal. By filtering the image, the contrast and equal processing are enhanced. According to the data characteristics of the pixel, the interference will interfere with various code operations. The background information is eliminated, and the characteristics of the rail surface damage, such as size, length, number, etc., are extracted. It even uses deep learning to mine the potential features of the data to classify and judge damage. The basic computer vision inspection system, the important part of the composition, includes the image acquisition module, the image-the preprocessing module, and the result judgment module. The research in computer vision also focuses on the optimization and exploration of these three modules. Usually, an inspection system is constructed by combining the improved methods of all stages. Liu and Wei presented a detection system based on image processing and edge detection to find the defect on the rail surface [41-44].

About improving the image acquisition module, it is mainly to adopt better equipment and optimize the method of acquiring images. The more creative research is that Francisco Javier de la Calle Herrero, etc. proposed a system that uses visual algorithm processing to find changes in pixel values between images [45]. Besides, Jia Ge et al. present an improved traditional system by combining a speed adaptive (SAA) system to adjust the appropriate exposure frequency and 
uniform image quality at any time according to the speed of the vehicle [46]. Guangyu Dai et al. studied an image processing system that is useful for online real-time detect the defects of the railway track surfaces. When the images are obtained by linear CMOS camera, the train detection speed can be $50 \mathrm{~km} / \mathrm{h}$ while the image is a planar array CMOS camera; the train speed can be $100 \mathrm{~km} / \mathrm{h}$ [47].

More researches focus on the image-processing module and the defect recognition module. The irregularity of rail surface defects makes automatic visual inspection very difficult. Because irregular defects appear randomly on the surface of the rail, the defects can only be located according to the image pixels. Moreover, in the natural environment, the uneven illumination and different reflectivity received by the curved surface of the rail will greatly impact the grayscale distribution of the image. The electronic interference in the image acquisition process will inevitably add some noise to the image [48]. At the same time, effective image enhancement and automatic threshold segmentation of defective images can improve detection efficiency. The image processing process mainly goes through the following steps. First: Track positioning, using the principle that the track surface and the background are quite different, such as edge detection, gray value positioning [49], etc. Second: Image noise reduction, the commonly used noise reduction methods are: mean filtering, median filtering, non-local means, Gaussian filtering, etc. Third: Image enhancement, traditional methods are global histogram equalization, adaptive histogram equalization, contrast-limited adaptive histogram equalization [50], local contrast enhancement [51], low-rank matrix decomposition, etc. At Last: Threshold segmentation, commonly used methods are: Ostu, Maximum between-class variance method, maximum entropy method, Iterative threshold method, etc.

The research on image processing has mainly improved and innovated on the original methods. Li et al. propose the Michelson-like contrast (MLC) measure image enhancement method with a new threshold algorithm, named proportion emphasized maximum entropy (PEME) [46]. Wu and Li optimized the background difference method by reducing the template preprocessing to reduce the influence of shadows and a loop threshold algorithm using histogram judgment [52]. Jinrui Gan et al. proposed a background-oriented defect detector (BODI), which uses modeling to determine specific image features of the track to distinguish background and defects to improve detection efficiency. The perfect detection rate of $100 \%$ for large defects, but only $41.78 \%$ for relatively small defects [53]. The defect-recognition rate reached $92.2 \%$. Yu et al. proposed a coarse-to-fine model (CTFM) to identify defects of different scales. Using different image processing methods to identify various defects has achieved better results than the previous method. The accuracy rate reached $100 \%$ in the actual evaluation [54] $\mathrm{Hu}$ et al. proposed an optical rail surface spalling detection algorithm based on visual saliency. Use a two-dimensional differential Gaussian (2D DoG) filter to reduce noise [55]. Wu Yunpeng et al. proposed the LWLCGSME model in a drone-based visual inspection system, using a new image 
enhancement algorithm based on local Weber-like contrast (LWLC) to enhance railway images and gray-scale stretched maximum entropy (GSME) segmentation images [56].

The image processing method of visual inspection shows better performance than other detection methods in detecting the rail surface peeling damage. However, in practical applications, it may be affected by more factors to reduce the accuracy. Therefore, some new image processing technologies have also been used. For example, Ashwani Kumar Dubey proposed using the maximally stable extremal region marking on the original system [57]. In the turnout surface wear detection field, Sysyn et al. introduced the application of computer vision in the diagnosis of common cross frogs [58]. However, technology has insufficient detection accuracy in detecting the rail surface cracks or more minor defects. With the continuous research of computer machine learning, the efficiency and accuracy of machine learning methods in image classification have surpassed the traditional classification methods. Since 2010, many scientific research institutions have conducted a lot of research on the categorization of rail surface defects in this field and make specific predictions about the development of cracks or defects. Machine learning methods are necessarily accompanied by defect assessment and prediction, so they are introduced in the next section.

\section{Classification Evaluation and Prediction}

Advances in technology have made it possible to automatically classify or evaluate the damage to the track surface, whether it is based on ultrasonic inspections or, of course, more visual images. Now the final evaluation and classification step almost have to rely on machine learning. Current methods for predicting features of track defects and even more minor cracks must also use machine learning. This section mainly introduces some research results in this field in the past ten years. Machine learning is divided into deep learning and shallow learning methods. The shallow machine learning calculation logic is relatively simple, and the classification analysis of available features is more accurate. Deep machine learning is more complex and has advantages in the classification and analysis of uncertain features.

\subsection{Shallow Machine Learning}

The traditional data classification method is a shallow machine-learning model. The basic process of using image information classification is as follows: First, image features are extracted. Commonly used image features include HOG (Histogram of Oriented Gradient) features [59], LBP (Local Binary Pattern) features [60], HAAR features, and SIFT (Scale Invariant Feature Transform) 
features [61]. After extracting the feature words, use the word bag model or clustering method (Marker controlled watershed segmentation (MCWS), K-means (KM) clustering, Expectation-Maximization (EM), and canny edge detection (CED), etc.) to reshape extracted features. Then, send the reshaped features to the classifier to get the classification result. Commonly used classifiers include support vector machines (SVM), decision trees, random forests, and Bayesian classification. Mercy et al. introduced three machine-learning methods for the prediction of track surface defects. Decision trees have the highest accuracy in predicting distortions and inhomogeneity. The random forest has the highest accuracy in predicting specific defects [62]. Regardless of accuracy, the recognition rate and complexity of the detection task. Machine learning has reached or even surpassed traditional methods.

Table 1 summarizes some research on the detection and judgment of track surface defects using image processing methods and the recently studied shallow machine learning methods. Shallow machine learning can already perform evaluation tasks other than the detection of rail surface defects, such as classification. Nearly $90 \%$ of the classification tasks of multi-class classification have been well applied in practice. With the research and development of deep learning, the effect of deep learning in target detection tasks surpasses all the above methods. And people gradually turn to the study of deep learning rail surface defect detection.

\subsection{Deep Machine Learning}

The deep learning model can learn deep abstract features layer by layer from the data set through supervised and unsupervised modes to achieve an abstract description of learning goals. The concept of deep learning has been produced since 1986. Deep learning models can learn deep abstract features layer by layer from the data set through supervised or unsupervised learning methods to achieve abstract descriptions of learning goals. In 2016, Hinton et al. elaborated on the deep learning system and proposed related technologies such as deep neural networks. In recent years, the wave of deep learning research has come and has produced many significant results.

The deep learning models currently used in the field of image and visual recognition mainly include Stacked auto-encoders, SAE, Deep belief network, DBN, and Convolutional Neural Networks, CNN), among which CNN has achieved satisfactory research and application results. A multi-layer convolutional neural network (convolutional neural network, CNN) can adaptively input data for feature learning and classify or recognize the learned features. CNN-based target detection is mainly divided into three categories: regression-based network models, such as YOLO, network models generated based on candidate regions, such as R-CNN, Fast R-CNN, Faster R-CNN; search-based network models, such as AlexNet; and VGGNet. Since deep learning simulates the human visual 
perception system, avoids the weaknesses of manual design features, and has the advantages of non-linearity and high parallelism, it has a wide range of applications.

Table 1

Image Processing and machine learning methods for rail defect detection

\begin{tabular}{|c|c|c|c|c|}
\hline Method & Features & Dataset & Results & Ref. \\
\hline Decision Tree & \multirow{2}{*}{$\begin{array}{c}\text { Rail surface } \\
\text { defect }\end{array}$} & \multirow{2}{*}{$\begin{array}{c}\text { Data from East } \\
\text { Coast Railway } \\
\text { division }\end{array}$} & Precision $=93.02 \%$ & \multirow{2}{*}[62]{} \\
\hline Random Forest & & & Precision $=95.23 \%$ & \\
\hline Region growing & \multirow{4}{*}{ The area of defect } & \multirow{4}{*}{$\begin{array}{l}\text { Rail Images } \\
\text { Acquisition }\end{array}$} & Sensitivity $=100 \%$ & \multirow{4}{*}[63]{} \\
\hline MCWS & & & Sensitivity $=90.9 \%$ & \\
\hline K-means & & & Sensitivity $=72.7 \%$ & \\
\hline Canny edge & & & Sensitivity $=81.8 \%$ & \\
\hline SVM & \multirow{4}{*}{ Crack } & \multirow{4}{*}{$\begin{array}{l}17000 \text { Rail images } \\
\text { from CRH and CM }\end{array}$} & Accuracy $=99.87 \%$ & \multirow{4}{*}[64]{} \\
\hline Random Forest & & & Accuracy $=100 \%$ & \\
\hline logistic regression & & & Accuracy $=99.74 \%$ & \\
\hline boosted tree & & & Accuracy $=99.92 \%$ & \\
\hline $\begin{array}{c}\text { AdaBoost } \\
\text { multiclassifier } \\
\text { +CART decision } \\
\text { tree }\end{array}$ & $\begin{array}{l}\text { Rail surface } \\
\text { defect classifier } \\
\text { recognition }\end{array}$ & 1200 rail images & $\begin{array}{c}\text { Recognition rate }(\%): \\
\text { Scale peeling } \\
\text { crack }=81.45 ; \text { Stripping } \\
\text { block }=79.64 ; \text { Tread } \\
\text { cracks }=82.27\end{array}$ & [65] \\
\hline Deep Forest & \begin{tabular}{|c|} 
Peeling and Crack \\
classification
\end{tabular} & $\begin{array}{l}\text { Video from X5 } \\
\text { PTZ camera }\end{array}$ & $\begin{array}{c}\text { Classification accuracy } \\
=100 \%\end{array}$ & {$[66]$} \\
\hline LWLC+GSME & $\begin{array}{l}25<\mathrm{T}-\mathrm{I} \text { Defect }< \\
\quad 255 \mathrm{~mm}^{2} ; \\
\text { T-II }>255 \mathrm{~mm}^{2}\end{array}$ & $\begin{array}{l}\text { HD video from } \\
\text { DJI Matrice } 600\end{array}$ & $\begin{array}{c}\text { T-I } \\
\text { Precision=88.63.22\%; } \\
\text { T-II Precision }=90.32 \%\end{array}$ & {$[56]$} \\
\hline GMM+MRF & $\begin{array}{c}\text { Rail surface } \\
\text { defect }\end{array}$ & $\begin{array}{c}\text { Image detection } \\
\text { system }\end{array}$ & $\begin{array}{c}\text { Precision }=88.8 \%, \text { recall } \\
=92.0 \%\end{array}$ & {$[67]$} \\
\hline $\begin{array}{c}\text { Bayesian } \\
\text { classification + EM }\end{array}$ & $\begin{array}{l}\text { Onboard detection } \\
\text { RUL prediction }\end{array}$ & $\begin{array}{c}\text { Data from 3D } \\
\text { acceleration } \\
\text { sensors }\end{array}$ & $\begin{array}{c}\text { RUL predicted accuracy }= \\
75 \%\end{array}$ & {$[68]$} \\
\hline $\begin{array}{l}\text { MODWPT+ Lasso } \\
\text { regression }\end{array}$ & $\begin{array}{c}\text { Trackside } \\
\text { detection } \\
\text { RUL prediction }\end{array}$ & $\begin{array}{l}\text { Data from 3D } \\
\text { acceleration } \\
\text { sensors }\end{array}$ & $\begin{array}{l}\text { RUL predicted accuracy }= \\
50 \%\end{array}$ & {$[69]$} \\
\hline
\end{tabular}

There have also been a lot of researches in the detection of rail surface defects. The surrounding set is the RSDD data released by Beijing Jiaotong University. By combining Wavelet Scattering and Neural Networks, Yang Jin improved classification accuracy up to $97.20 \%$ and $94.74 \%$ for the two datasets [70]. 
Combining the advantages of the bilateral full convolutional network and CRF proposed by Zhang Ziwen and others will also significantly improve detection accuracy [71]. This article introduces the use of deep learning, mainly neural convolution, to detect rail surface defects in the past ten years. Part of the research on classification and evaluation is shown in Table 2. Various methods can be intuitively compared.

\subsection{Prediction}

The losses caused by the catastrophic railway failures caused by RCF are difficult to estimate. Although the rails are very expensive, the defects are found in time, and the prediction and evaluation are carried out. The forecast reduces the number of inspections and avoids disasters, which is an economical and safe method. In the field of state prediction, many methods of finite element analysis are used. For example, H. M. El-Sayed establishes a wheel-rail stress model, simulates the rail head's damage state through finite element analysis, and predicts the service life of the rail through long-term data [72]. The ability of machine learning in the field of prediction has also been proven to have more potential. In recent years, there have been more studies for the prediction of rail damage. Ahmed Lasisi et al. proposed an integrated learning framework. It takes advantage of the advantages of a single machine learning tools and overcomes assuming basic distribution (such as Weibull) to predict the deviation of the defect, and also consider the railway characteristics except for the traffic volume (Mt) to achieve the purpose of optimizing the prediction results [73]. Sysyn's research team conducted two studies on the remaining useful life (RUL) prognosis of common crossings as shown in Table 2 on the inspection data obtained by the on-side and the onboard intrinsic measurement system of the turnout.

The two machine learning methods proposed in the research systematically carry out the processes of feature extraction, selection, fusion, and degradation modeling and are then used to deal with the problem. It corresponds to about 50\% and $75 \%$ of the condition indicator reached. Therefore, the available period would be sufficient for maintenance planning, thus avoiding operational hindrance costs [66].

Table 2

Deep learning detection methods

\begin{tabular}{|l|l|l|l|l|}
\hline Method & Features & Dataset & Results & Ref. \\
\hline DCNN & $\begin{array}{l}\text { lassification: Weld, } \\
\text { large, middle and } \\
\text { small Squat; Joint }\end{array}$ & $\begin{array}{l}22408 \text { images; size 100 } \\
\times 50\end{array}$ & Accuracy=92\% & {$[74]$} \\
\hline CNN & Defect on Surface & $\begin{array}{l}25.000 \text { profiles per } \\
\text { second }\end{array}$ & Accuracy=98\% & {$[75]$} \\
\hline CNN & Defect on Surface & $\begin{array}{l}7897 \text { images size } \\
4096 \times 3000\end{array}$ & $\begin{array}{l}\text { Precision=61.14\%; } \\
\text { Recall=75.52\% }\end{array}$ & {$[76]$} \\
\hline
\end{tabular}




\begin{tabular}{|c|c|c|c|c|}
\hline \begin{tabular}{|l} 
Cropped \\
CNN
\end{tabular} & Rail Surface location & $\begin{array}{l}5793 \text { images; size } 960 \\
\times 1280\end{array}$ & $\begin{array}{l}\text { Recall }=92.54 \% ; \text { Precisio } \\
n=92.8 \%\end{array}$ & [77] \\
\hline MOLO & $\begin{array}{l}\text { Classification: } \\
\text { corrugation, fatigue } \\
\text { block, stripping } \\
\text { off block }\end{array}$ & $\begin{array}{l}96944 \text { images; } \\
\text { size } 224 \times 224 \times 3\end{array}$ & $\begin{array}{l}\text { Corrugation } \\
\text { AP=95.28\%;Fatigue } \\
\text { block AP=84.60\%; } \\
\text { Stripping } \\
\text { off block AP=82.33\% }\end{array}$ & [78] \\
\hline SegNet & Rail surface defects & $\begin{array}{l}120 \text { rail training } \\
\text { images; } \\
\text { size } 1250 \times 55\end{array}$ & Detection Rate $=100 \%$ & [79] \\
\hline YOLOv3 & Rail surface defect & $\begin{array}{l}\text { 184images; size } 416 \times \\
416\end{array}$ & $\begin{array}{l}\text { Recognition rate more } \\
\text { than } 97 \%\end{array}$ & [80] \\
\hline $\begin{array}{l}\text { MRF- } \\
\text { GMM and } \\
\text { CNN }\end{array}$ & d Rail surface defects & $\begin{array}{l}6 \text { categories, } 2700 \\
\text { samples; size } 250 \times \\
160\end{array}$ & $\begin{array}{l}\text { Precision }=96.74 \%, \\
\text { Recall }=94.13 \% \\
\text { Overlap }=95.18 \%\end{array}$ & [81] \\
\hline DCNN & $\begin{array}{l}\text { Detection and } \\
\text { classification of } \\
\text { abrasion scar crack } \\
\text { corrugation }\end{array}$ & $\begin{array}{l}38000 \text { training images; } \\
\text { size } 512 \times 512\end{array}$ & $\begin{array}{l}\text { The accuracy of defect } \\
\text { classification achieves } \\
96.55 \%\end{array}$ & [82] \\
\hline $\begin{array}{|ll|}\text { Faster } & \mathrm{R}- \\
\text { CNN } & \\
\end{array}$ & Rail surface defect & $\begin{array}{l}1000 \text { images in } \\
\text { environments }\end{array}$ & $\begin{array}{l}\text { Average Precision } \\
=97.8 \%\end{array}$ & [83] \\
\hline \begin{tabular}{|l|} 
OC-IAN \& \\
OC-TD
\end{tabular} & Rail surface defect & RSDDs & $\begin{array}{l}\text { T-I: pre }=84.21 \% \text {; } \\
\text { T-II: pre. }=91.76 \%\end{array}$ & [84] \\
\hline $\begin{array}{l}\text { RBGNet \& } \\
\text { LWLC-ME } \\
\end{array}$ & Rail surface defect & $\begin{array}{l}\text { video images resized to } \\
1280 \times 720\end{array}$ & Precision over $90 \%$ & [85] \\
\hline Mcnet & Rail surface defect & $\begin{array}{l}3936 \text { NRSD images, } \\
\text { size } 400 \times 400\end{array}$ & $\begin{array}{l}\text { Manmade Pre }=85.28 \% \\
\text { Natural Pre. }=72.74 \%\end{array}$ & [86] \\
\hline
\end{tabular}

\section{Results and Discussion}

In this article, various methods for detecting railway surface defects are discussed. The detection of defects on the rail surface is challenging because the cracks have an irregular form and have no specific shape or size. Each detection method may have some advantages and some disadvantages. However, visual inspection and electromagnetic technologies such as ACFM and MFL have significant benefits in comprehensively comparing inspection efficiency and accuracy. In terms of detecting speed, both methods can be used to obtain data on a running train. However, the sensitivity of electromagnetic technology at high speeds is not high. ACFM is generally less sensitive to short or shallower discontinuities than traditional eddy currents. MFL cannot detect relatively small defects. From the distribution of the number of papers, it can also be shown that more scholars are engaged in the research of visual inspection, especially the visual inspection direction in machine learning, on rail surface defects. 
The visual inspection method of image processing has been well certified in the laboratory, but in the actual environment, there is a lot of external interference, different texture characteristics, changing lighting conditions, and stains on the rails, etc., will cause the image processing process significant interference reduces the accuracy of detection. The shallow machine learning methods shown in Table 1 have achieved $90 \%$ or more accuracy in defect detection. Even for some major defect categories, the accuracy can reach $80 \%$. The deep machine learning shown in Table 2 further strengthens this effect. The detection accuracy is further improved by $5-7 \%$. Many neural convolution processes are combined with other image processing or SVM, random forest, and other machine learning methods to achieve better detection results. Many methods have proved the feasibility and relative accuracy in real track surface damage detection. Enlarging the data set can avoid overfitting of the model and enhance the accuracy of detection. But larger data sets require more data collection time and investment, and deep machine learning requires a lot of computing time in working hours and also has high requirements for computing equipment. The method of deep machine learning in the classification of rail surface damage has shown considerable prospects. There are still very few studies used to predict the surface defect of railroad tracks, and the prediction results are also relatively unstable. The primary research method does not use deep machine learning because shallow machine learning methods are easier to control the number and focus of features.

\section{Conclusions}

This article focuses on reviewing the most advanced rail surface damage detection methods in the past decade. These results have been published in top journals and conferences. We reviewed the criteria of the 70 research articles screened after the application and checked their content in detail. These articles are evaluated based on the detection methods they use, performance results, and limitations. Through analysis, we can infer that machine learning visual inspection methods can be widely used to detect railway surface defects. Combining multiple methods with sub-gradient detection of different types of defects has a better detection effect. More research needs to focus on the detection of rail surface cracks. At present, satisfactory results have been achieved for spalling and squats, while relatively small surface crack research has hardly made significant progress. For the reasonably complicated in 3 classifications task, orbital surface defect classification accuracy can reach $75 \%$ or even $85 \%$. After more data sets and more research results to overcome false defects or even minor defects are proposed in the future, the accuracy of classification will be further improved. In the future, research on crack damage detection on the surface of the steel rail will mainly focus on the optimization and improvement of deep machine learning, especially in the field of CNN. The research on detection can be extended in the direction of evaluating the defects and then predicting the service life of the rail. More image processing methods suitable for real environments are also necessary. 


\section{References}

[1] A. Németh, S. Fischer, Investigation of glued insulated rail joints with special fiber-glass reinforced synthetic fishplates using in continuously welded tracks. Pollack Periodica, Vol. 13, No. 2, 2018, pp. 77-86

[2] A. Németh, S. Fischer, Investigation of glued insulated rail joints applied to CWR tracks. Facta Universitatis Mechanical Engineering, 2021, 7642

[3] S. Fischer, Investigation of effect of water content on railway granular supplementary layers. Naukovyi Visnyk Natsionalnoho Hirnychoho Universytetu, No. 3, 2021, pp. 64-68

[4] Blagojević, Aleksandar, Željko Stević , Dragan Marinković , Sandra Kasalica, and Snježana Rajilić. "A Novel Entropy-Fuzzy PIPRECIA-DEA Model for Safety Evaluation of Railway Traffic." MDPI Symmetry, 2020

[5] Wikipedia. List of countries by rail transport network size. 2017. https://en.wikipedia.org/wiki/List_of_countries_by_rail_transport_network _size

[6] Popvic, Zdenka, V. Radovic, Luka Lazarevic, and G. Tepic. "Rail inspection of RCF defects." Metalurgia-Sisak, Oct 2013, pp. 537-540

[7] Ovchinnikov, Dmitry, Alexey Bondarenko, Lei Kou, and Mykola Sysyn. "Extending service life of rails in the case of rail head defect." Gradevinar, 2 2021, pp. 119-125

[8] Falamarzi, Amir, Sara Moridpour, and Majidreza Nazem. "Areview on Existing Sensors and Devices for Inspecting Ralway Infrastructure." Jurnal Kejuruteraan, 2019, pp. 1-10

[9] Oliveira, H. and Correia, P. L. "Automatic road crack detection and characterization." IEEE Transactions on Intelligent, 2012, pp. 155-168

[10] Marais, J J, and K C. Mistry. "Rail integrity management by means of ultrasonic testing." FFEMS, 2010, pp. 931-938

[11] Shang, Lidan, Qiushi Yang, Jianing Wang, Shubin Li, and Weimin Lei. "Detection of rail surface defects based on CNN image recognition and classification." International Conference on Advanced Communication Technology. IEEE, 2018

[12] Cannon, D. F., K. O. Edel, S. L. Grassie, and K. Sawley. "Rail defects: an overview." Fatigue Fract ENgng Mater Struct, 2003, pp. 865-887

[13] Li, Q Y, and S W Ren. "A visual detection system for rail surface defects." IEEE Transactions on Systm, 2012, pp. 1531-1542

[14] Lu, Z W. "Overall comments on track technology of high speed railway." Journal of Railway Engineering Society, 2007, pp. 41-54 
[15] Kurhan Dmytro and Maksym Havrylov. "The Mathematical Support of Machine Surfacing for the Railway Track." Acta Technica Jaurinensis, 2020, pp. 246-267

[16] Ye, Jiaqi, Edward Stewart, and Clive Roberts. "Use of a 3D model to improve the performance of laser-based railway track inspection." Proc IMechE Part F: J Rail and Rapid Transit, Aug 2018

[17] Rikhotso, Vonani, Nico Steyn, and Yskandar Hamam. "3D Rail Modelling and Measurement for Rail Profile Condition Assessment." IEEE Africon 2017 Proceedings, 2017, pp. 1522-1527

[18] Jessop, Casey, Johan Ahlstrom, Lars Hammar, Soren Faester, and Hilmar K. Danielsen. "3D characterization of rolling contact fatigue crack networks." Wear, 2016, pp. 293-400

[19] Naeimi, meysam, et al. "Reconstruction of the rolling contact fatigue cracks in rails using X-ray computed tomography." NDT and E International Elsevier Ltd, 2017, pp. 199-212

[20] Niu, Menghui, et al. "Unsupervised saliency detection of rail surface defects using stereoscopic images." Transactions on Informatics, 2021, pp. 2271-2281

[21] Sysyn, Mykola, Kluge Franziska, Gruen Dimitri, Vitalii Kovalchuk, and Olga Nabochenko. "Experimental analysis of rail contact fatigue damage on frog rail of fixed common crossing 1:12." Journal of Failure Analysis and Prevention, 2019, pp. 1077-1092

[22] Bentoumi, M, and Pbloch G. Aknin. "online rail defect diagnosis with differential eddy current probes and specific detection processing." The European Phzsical Journal Applied Physics, 2003, pp. 227-233

[23] Pohl, R, and R Krull. "A new eddy current instrument in a grinding train." In Proceeding of the ECNDT, 2006, pp. 178-184

[24] Thomas, H, and Thanspach G $\mathrm{G}$ Heckel. "Advantage od a combined ultrasonic and eddy current examination for railway inspection trains." Insight-Non-destructive Testibf abd Condition Monitoring, 2007, pp. 341344

[25] Huang, F Z, D H Gao, and X H Ding. " The Design and Application of the Card-type Eddy Current Sensor for the Detection of the Surface Defects of the Switch Rail." Nondestructive Testing, 2014, pp. 63-68

[26] Wang, Ping, Yunlai Gao, Guiyun Tian, and et al. "Velocity effect analysus of dynamic magnetuyation in high speed magnetic flux leakage inspection." NDT \& E International, 2014, pp. 7-12

[27] Jia, Yinliang, Kangwu Liang, Ping Wang, Kailun Ji, and Peng Xu. "Enhancement method of magnetic flux leakage signals for rail track 
surface defect detection." IET Science, Measurement \& Technology, 2020, pp. 711-718

[28] Ji, Kailu, Ping Wang, Yinliang Jia, Yunfei Ye, and ShunziI Ding. "Adaptive Filtering Method of MFL Signal on Rail Top Surface Defect Detection." IEEE Access, 2021, pp. 87351-87360

[29] Hamed, Rowshandel and L Nicholson Gemma, et al. "An integrated robotic system." journal of rail and rapid transit, 2013, pp. 310-322

[30] Chacón Muñoz, J. M., F. P. García Márquez, and M. Papaelias. "Railroad inspection based on ACFM employing a non-uniform B-spline approach." 2013, pp. 605-617

[31] Lugg, Martin, and Mayorkinos P Papaelias. "Detection and evaluation of rail surface defects using alternating current field measurement techniques." journal of rail and rapid transit, 2012, pp. 530-542

[32] Li, Zili, Maria Molodova, Alfredo Núñez, Senior Member, and Rolf Dollevoet. "Improvements in Axle Box Acceleration Measurements for the Detection of Light Squats in Railway Infrastructure." Transactions on Industrial electronics, 2015, pp. 4385-4399

[33] Keong Ng, Andrew, Landong Martua, and George Sun. "Dynamic Modelling and Acceleration Signal Analysis of Rail Surface Defects for Enhanced Rail Condition Monitoring and Diagnosis." The $4^{\text {th }}$ International Conference on Intelligent Transportation Engineering. Sep. 2019

[34] Sysyn, Mykola, Dimitri Gruen, Ulf Gerber, Olga Nabochenko, and Vitalii Kovalchuk. "Turnout Monitoring with Vehicle Based Inertial Measurements of Operational Trains: A Machine Learning Approach." Communications, 2019, pp. 42-49

[35] Sysyn, Mykola, Olga Nabochenko, Franziska Kluge, Vitalii Kovalchuk, and Andriy Pentsak. "Common Crossing Structural Health Analysis with Track-Side Monitoring." COMMUNICATION, 2019, pp. 77-84

[36] Jiang, Yi, et al. "Non-contact ultrasonic detection of rail surface defects in different depths." Proceedings of Front 2018. 2018

[37] Zhang, Hui, et al. "Wavenumber Imaging of Near-Surface Defects in Rails using Green's Function Reconstruction of Ultrasonic Diffuse Fields." Sensors, April 2019

[38] Zhang, Yuehong, Lin Luo, Yu Zhang, Xiaorong Gao, and Jiang Long. "Interlaced scanning by laser ultrasonic for defects imaging of train rail surface." Eleventh International Conference on Information Optics and. Xi an, 2019

[39] Ramzan, Bilawal, Sohail Malik, S. M. Ahmad, and Milena Martarelli. "Railroads Surface Crack detection using Active Thermography." 2021, pp. 183-198 
[40] Zhuravlev, Andrey, Vladimir Razevig, Sergey Ivashov, Aleksey Skrebkov, and Viktor Alekseev. "On the Use of Microwave Holography to Detect Surface Defects of Rails and Measure the Rail Profile." sensors, 2019

[41] Liu, Yuxin, and Xiukun Wei. "Track Surface Defect Detection Based on Image Processing." Proceedings of the $3^{\text {rd }}$ International Conference on Electrical. Singapore, 2018, pp. 255-232

[42] Fu, Shengwen, and Zhanjun Jiang. "Research on Image-based Detection and Recognition Technologies for Cracks on Rail Surface." 2019 International Conference on Robots \& Intelligent System (ICRIS) Lan Zhou, 2019, pp. 98-102

[43] Wu , Yunpeng, Yong Qin, and Limin Jia. "Research on Rail Surface Defect Detection Method Based on UAV Images." 2018 Prognostics and System Health Management Conference. 2018, pp. 553-559

[44] Min, Yongzhi, Benyu Xiao, Jianwu Dang, Biao Yue, and Tiandong Cheng. "Real time detection system for rail surface defects based on machine vision." 1. EURASIP Journal on Image and Video Processing, 2018

[45] Herrero, Francisco Javier de la Calle, Daniel F. García, and Rubén Usamentiaga. "Inspection System for Rail Surfaces Using Differential Images." IEEE Transactions on Industry Applications, 2018, pp. 4948-4958

[46] Li, Qingyong, Shengwei Ren, Member, and IEEE. "A Visual Detection System for Rail Surface Defects." IEEE Transactions on Systems, 2012, pp. $1531-1543$

[47] Dai , Guangyu, et al. "Online real-time detection method for defects of railroad track surface." Applied Mechanics and Materials, 2013, pp. 10171020

[48] Tang, Bo, Jianyi Kong, and Shiqian Wu. "Overview of surface defect detection based on machine." Chinese Journal of image and graphics, 2017

[49] Zhao, Kang, Laizhen Luo, Zhengmin Ren, and Qingchen Fu. "A surface defect detection system for railway track based on machine vision." MCTE. 2020

[50] Mohan, S., and M. Ravishankar. "Optimized histogramm based contrast limited enhancement for mammogram." American Council for an Energzefficiept Economy Intternational Journal on Information Technology, 2013, pp. 66-71

[51] Gordon, R., and Rangayan. "Feature enhancement of film mammogram using fixed and adaptive neighborhoods." Applied Optic, 1984, pp. 560-564

[52] Wu, Yidi, and Lin Li. "Inspection of Rail Surface Defects Image Based on Histogram Processing by the Judgment Threshold." Proceedings of 2018 Chinese Intelligent Systems Conference. Singapore, 2019, pp. 199-209 
[53] Gan, Jinrui and Jianzhu Wang, Haomin Yu, Qingyong Li, and Zhiping Shi. "Online Rail Surface Inspection Utilizing Spatial Consistency and Continuity." IEEE Transactions on Systems, Man, and Cybernetics: Systems, 2018, pp. 2741-2751

[54] Yu, Haomin, et al. " A coarse-to-fine model for rail surface defect detection." IEEE Transactions on Instrumentation and Measurement, 2018, pp. 656-666

[55] Hu, Zhixin, Hongtao Zhu, Ming Hu, and Yong Ma. "Rail Surface Spalling Detection Based on Visual Saliency." IEEJ Transactions on Electrical and Electronic Engineering, 2018, pp. 505-509

[56] Wu, Yunpeng, Yong Qin, Zhipeng Wang, and Limin Jia. "A UAV-Based Visual Inspection Method for Rail Surface Defects." applied sciences, 2018

[57] Dubey, Ashwani Kumar, and Zainul Abdin Jaffery. "Maximally Stable Extremal Region Marking) based Railway Track Surface Defect Sensing." IEEE Sensors, 2016, pp. 9047-9052

[58] Sysyn, Mykola, Ulf Gerber, Olga Nabochenko, Dmitri Gruen, and Franziska Kluge. "Prediction of Rail Contact Fatigue on Crossings Using ImageProcessing and Machine Learning Methods." Urban Rail Transit, 2019, pp. 123-132

[59] Dalal, N, and B Triggs. "Histograms of oriented gradients for human detection." IEEE Computer Society, 2005

[60] Rodriguez, Y, and S Marcel. "Face authentication using adapted local binary pattern histograms." Springer Verlag, 2006, pp. 321-332

[61] Mercy, K. Grace, and Sri. K. Srinivasa Rao. "A Framework for Rail Surface Defect Prediction Using Machine Learning Algorithms." Proceedings of the International Conference on Inventive Research in Computing Applications (ICIRCA 2018) IEEE Xplore, 2018, pp. 972-978

[62] Jaffery, Zainul Abdin, Nadeem Ahmad, and Deependra Sharma. "Performance comparison of segmentation techniques for detection of defect in rail head surface images." 2017, pp. 132-137

[63] Wang, Long, Li Zhuang, and Zijun Zhang. "Automatic Detection of Rail Surface Cracks with a Superpixel-Based Data-Driven Framework." J. Comput. Civ. Eng, 2019

[64] Yue, Biao, Yangping Wang, Yongzhi Min, Zhenhai Zhang, Wenrun Wang, and Jiu Yong. "Rail Surface Defect Recognition Method Based on AdaBoost Multi-classifier Combination." Proceedings of APSIPA Annual Summit and Conference 2019, LanZhou, 2019

[65] Long Wang; Li Zhuang; and Zijun Zhang. "Rail Surface Defect Recognition and Classification Method Based on Deep Forest." EITRT 2019, 2019, pp. 493-503 
[66] Zhang, Hui, Xiating Jin, Q. M. Jonathan Wu, Yaonan Wang, Zhendong He, and Yimin Yang. "Automatic Visual Detection System of Railway Surface Defects With Curvature Filter and Improved Gaussian Mixture Model IEEE Transactions on Instrumentation and Measurement, 2018, pp. 15931608

[67] Sysyn, M., U. Gerber, F. Klug, O. Nabochenko, and V. Kovalchuk. "Turnout remaining useful life prognosis by means of on-board inertial measurements on operational trains." International Journal of Rail Transportation, 2019, pp. 1-23

[68] Sysyn, Mykola. "Improvement of inspection system for common crossings by track side monitoring and prognostics." Structural Monitoring and Maintenance, 2019, pp. 219-235

[69] Lowe, D G. "Distinctive Image Features from Scale Invariant Keypoints." International Journal of Computer Vision, 2004, pp. 91-110

[70] Jin, Yang. "Wavelet Scattering and Neural Networks for Railhead Defect Identification." Materials, 2021

[71] Zhang, Ziwen, Mangui Liang, and Zhe Wang. "A Deep Extractor for Visual Rail Surface Inspection." IEEE Access, 2021, pp. 21798-21801

[72] El-sayed, H. M., M. Lotfy, H. N. El-din Zohny, and H.S. Riad. "Prediction of fatigue crack initiation life in railheads using finite elementanalysis." Ain Shams Engineering Journal, 2018, pp. 2329-2342

[73] Lasisi, Ahmed, and Nii Attoh-Okine. "Machine Learning Ensembles and Rail Defects Prediction: Multilayer Stacking Methodology." Journal of Risk and Uncertainty, 2019

[74] Faghih-Roohi, Shahrzad, Siamak Hajizadeh, Alfredo Nu'nez , Robert Babuska, and Bart De Schutter. "Deep Convolutional Neural Networks for Detection of Rail Surface Defects." 2016 International Joint Conference on Neural Networks (IJCNN) IEEE, 2016, pp. 2585-2591

[75] Santur, Yunus, Mehmet Karaköse, and Erhan Akın. "A New Rail Inspection Method Based on Deep Learning Using Laser Cameras." IEEE, 2017

[76] García, Daniel F., Iván García, Francisco J. delaCalle, and Rubén Usamentiaga. "A Configuration Approach for Convolutional Neural Networks used for Defect Detection on Surfaces." $20185^{\text {th }}$ International Conference on Mathematics and Computers in Sciences and Industry (MCSI) 2018, pp. 44-52

[77] Shang, Lidan, Qiushi Yang, Jianing Wang, Shubin Li, and Weimin Lei. "Detection of Rail Surface Defects Based on CNN Image Recognition and Classification." International Conference on Advanced Communications Technology(ICACT) 2018 
[78] Yuan, Hao, Hao Chen, ShiWang Liu, Jun Lin, and Xiao Luo. "A deep convolutional neural network for detection of rail surface defect." IEEE Xplore, 2020

[79] Liang, Zhicong, Hui Zhang, Li Liu, Zhendong He, and Kai Zheng. "Defect Detection of Rail Surface with Deep Convolutional Neural Networks." Proceeding of the $201813^{\text {th }}$ World Congress on Intelligent Control and Automation. Chang Sha: IEEE, 2018

[80] Song, Yanan, Hui Zhang, Li Liu, and Hang Zhong. "Rail Surface Defect Detection Method Based on YOLOv3 Deep Learning Networks." IEEE, 2018, pp. 1564-1572

[81] Jin, Xiating, et al. "DM-RIS: Deep Multimodel Rail Inspection System With Improved MRF-GMM and CNN." IEEE Transactions on Instrumentation and Measurement, 2020, pp. 1051-1066

[82] Li, Xiaoqing, Ying Zhou, and Hu Chen. "Rail Surface Defect Detection Based on Deep Learning." Eleventh International Conference on Graphics and Image Processing. Hang Zhou, 2019

[83] Chen, Xiaobo, and Huimin Zhang. "Rail Surface Defects Detection Based on Faster RCNN." 2020 International Conference on Artificial Intelligence and Electromechanical Automation (AIEA) IEEE Xplore, 2020

[84] Zhang, Defu, Kechen Song, Qi Wang, Yu He, Xin Wen, and Yunhui Yan. "Two Deep Learning Networks for Rail Surface Defect Inspection of Limited Samples With Line-Level Label." IEEE Transactions on Industrial Informatics, 2021, pp. 6731-6742

[85] Wu, Yunpeng, and Yong Qin, et al."Hybrid deep learning architecture for rail surface segmentation and surface defect detection." Computer-Aided Civil and Infrastructure Engineering, 2021, pp. 1-18

[86] Zhang, Defu, Kechen Song, Jing Xu, Yu He, Menghui Niu, and Yunhui Yan. "MCnet: Multiple Context Information Segmentation Network of NoService Rail Surface Defects." IEEE Transactions on Instrumentation and Measurement, 2021 